\title{
Microtubules and motor proteins: Mechanically regulated self-organization in vivo
}

\author{
S.K. Vogel ${ }^{1}$, N. Pavin ${ }^{2,3}$, N. Maghelli ${ }^{1}$, F. Jülicher ${ }^{2}$, and I.M. Tolić-Nørrelykke ${ }^{1, a}$ \\ 1 Max-Planck-Institute of Molecular Cell Biology and Genetics, 01307 Dresden, Germany \\ 2 Max-Planck-Institute for the Physics of Complex Systems, 01187 Dresden, Germany \\ 3 Department of Physics, Faculty of Science, University of Zagreb, 10002 Zagreb, Croatia
}

\begin{abstract}
A key aspect of life is sexual reproduction, which requires concerted movement. For successful mixing of the genetic material, molecular motors move the nucleus back and forth inside the cell. How motors work together to produce these large-scale movements, however, remains a mystery. To answer this question, we studied nuclear movement in fission yeast, which is driven by motor proteins pulling on microtubules. We show that motor proteins dynamically redistribute from one part of the cell to the other, generating asymmetric patterns of motors and, consequently, of forces that generate movement. By combining quantitative live cell imaging and laser ablation with a theoretical model, we find that this dynamic motor redistribution occurs purely as a result of changes in the mechanical strain sensed by the motor proteins. Our work therefore demonstrates that spatio-temporal pattern formation within a cell can occur as a result of mechanical cues (Vogel et al., 2009), which differs from conventional molecular signaling, as well as from self-organization based on a combination of biochemical reactions and diffusion.
\end{abstract}

\section{Introduction}

Molecular motors exert force on microtubules to position nuclei, spindles, and other organelles in eukaryotic cells [1-4]. Much is known about the behavior of individual molecular motors in vitro [5]. In vivo, however, a large number of motors act together. A key question is how a multitude of motors and microtubules organize their behavior into a concerted movement in a living cell.

Oscillations of organelles, whereby subcellular bodies exhibit large scale motion moving back and forth, are generated by a multitude of motors. Such spontaneous oscillations represent a simple example of complex dynamics and are ideally suited for studying the dynamic properties of a multitude of motors and microtubules. An understanding of motor coordination can be gained from the features inherent to the oscillatory behavior, such as the switch in direction of motion and the interplay between opposing forces, typically acting simultaneously. Spontaneous oscillation of the nucleus, spindle, and chromosomes have been observed in a number of eukaryotic systems [3,6-9]. Theoretical descriptions for motor-based spindle and chromosome oscillations have been introduced [10-15]. These proposed scenarios rely on detachment of the force generators from microtubules in response to load forces, consistent with in vitro studies [16]. This assumption, however, was not directly verified experimentally in vivo. Furthermore, all models consider force generators that are immobilized at the cell cortex or on the

a e-mail: tolic@mpi-cbg.de 


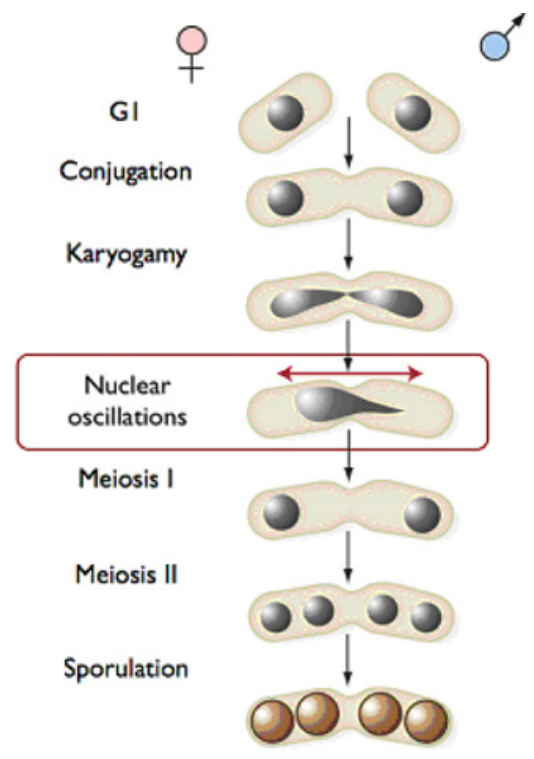

Fig. 1. Sexual reproduction of fission yeast cells.

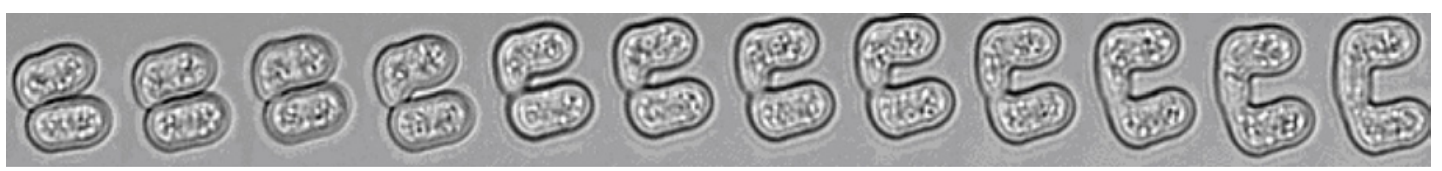

Fig. 2. A pair of yeast cells during mating. Nuclear oscillations begin when the zygote is formed and the two nuclei fuse (karyogamy). The images are acquired over a period of $\sim 5$ hours.

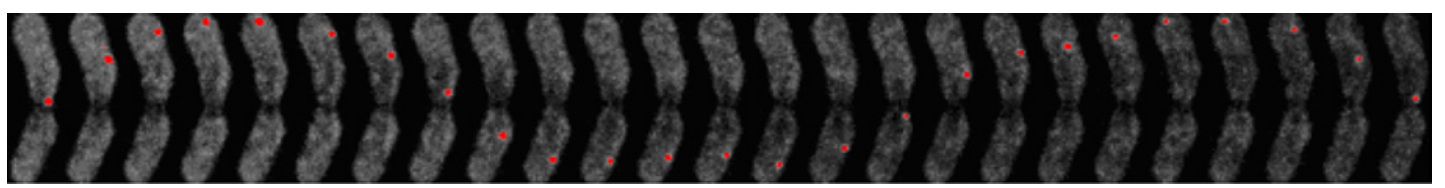

Fig. 3. Oscillations of the spindle pole body (SPB). The SPB was tagged with green fluorescent protein (GFP) and is here shown in red. The period of the oscillations is $\sim 5$ minutes, and the cell length is $14 \mu \mathrm{m}$.

chromosomes. However, the spatial distribution of force generators in these systems, as well as the dynamics of these distributions, have not been directly observed.

The nuclear oscillations in the meiotic prophase of the fission yeast Schizosaccharomyces pombe provide an excellent system to study oscillatory movement, which depends on cytoplasmic dynein and microtubules [17]. These oscillations occur after two cells of opposite mating types conjugate together, and their nuclei fuse into one (Figs. 1 and 2). Fluorescently marked dynein and microtubules can be observed during the whole process. When oscillating, the nucleus moves from one end of the cell to the other. Its motion spans the entire $14 \mu \mathrm{m}$ length of the cell and has a period of 5-10 minutes, and lasts for several hours [7]. The meiotic nuclear oscillations are crucial for proper chromosome pairing, recombination, and spore viability $[17,18]$. Similar chromosome movements have been observed in meiotic prophase in a variety of model organisms, from budding yeast to mouse $[19,20]$, and the role of these movements in chromosome pairing and recombination has been demonstrated [24].

The nuclear oscillations in $S$. pombe are led by the motion of the spindle pole body (SPB, a centrosome equivalent in yeast; Fig. 3) [7]. SPB movement depends on microtubules [21], which extend from the SPB towards the two ends of the cell (Fig. 4). The microtubule minus-ends are at the SPB, whereas the plus-ends point towards the cell periphery [22]. We refer to the 


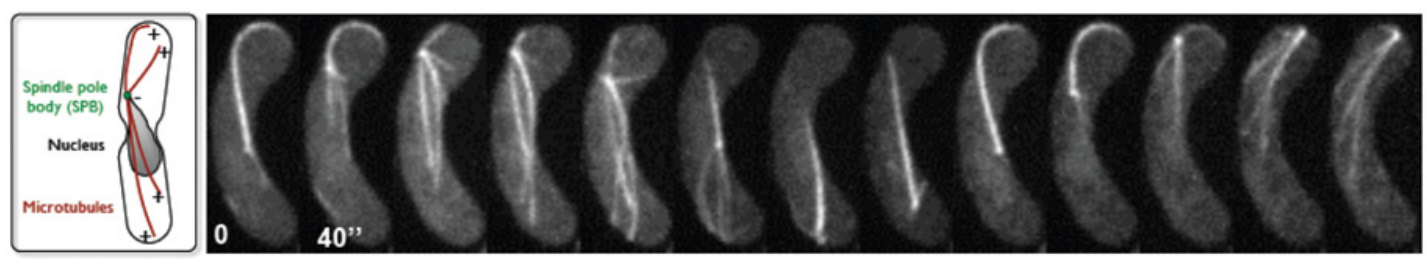

Fig. 4. Microtubules (MTs) during the oscillations. Schematic drawing of a yeast cell during meiotic oscillations, together with time-lapse images of oscillations in a cell with GFP-labeled MTs (white lines) and the GFP-labeled SPB (bright white spot).

microtubules extending from the SPB in the direction of the SPB movement and in the opposite direction as the leading and trailing microtubules, respectively. The leading microtubules are found in close proximity to the cell cortex [22]. Only parts of the length of the trailing microtubules are close to the cortex. Microtubule catastrophe, i.e., the transition from growth to shrinkage, occurs predominantly when the microtubule tip is at the cell end [22]. As the SPB moves, the leading microtubules typically shrink, while the trailing ones grow [22]. While these observations suggest that MT growth and shrinkage may be linked to nuclear oscillations, the physical mechanisms underlying the oscillations remained unclear.

By combining quantitative live cell imaging and laser ablation with a new theoretical model describing the motor-microtubule system, we show that self-organized dynamic redistribution of dynein motors can account for the observed nuclear oscillations. We show that dynein motors, responding to load forces, cooperatively detach from the trailing microtubules. After redistributing via the cytoplasm, they attach along the leading microtubules, thereby generating the force asymmetry necessary for the oscillations.

\section{Results}

\subsection{Pulling forces are exerted along the microtubules}

We started dissecting the mechanism of SPB oscillations by asking where the force that drives SPB movement is generated. Based on the known microtubule polarity with minus-ends located at the SPB, there are three possibilities. First, dynein motors immobilized at the cell cortex walk towards the minus-ends of the leading microtubule, thereby pulling the microtubule and the SPB towards the cortex [22]. Second, dynein motors immobilized at the SPB move towards the growing minus-end of the trailing microtubule. This movement pushes the trailing microtubule away from the SPB and, upon its contact with the cortex at the cell tip, the microtubule pushes the SPB forward. In the third scenario, the force exerted by microtubule polymerization at the plus-end against the cortex at the cell tip pushes the SPB forward [21].

Experiments in which a photo-bleached region of a GFP-labeled microtubule was observed to be stationary with respect to the SPB suggest that microtubules do not polymerize at their minus-ends [22], thus excluding the second model. The two remaining models can be distinguished by mechanical perturbations of the system using laser ablation of specific microtubules. Both models lead to testable predictions. If the SPB movement is mainly driven by pulling, then ablation of the leading microtubules will disturb the movement, whereas ablation of the trailing microtubules will not affect the movement. In contrast, if the SPB movement is driven mainly by pushing, then the ablation will have an opposite effect. Using laser ablation of specific microtubules $[23,25,26]$, we perturbed the force balance in the cell to directly test the contribution of pulling and pushing. Our experiments showed that after selective ablation of the leading MTs, the SPB rapidly changed the direction of its motion (Fig. 5). On the contrary, ablation of trailing microtubules did not affect the movement (Fig. 5). These data provide direct evidence that SPB movement is driven by pulling via the leading microtubules [22], while the contribution of pushing is negligible [27]. 

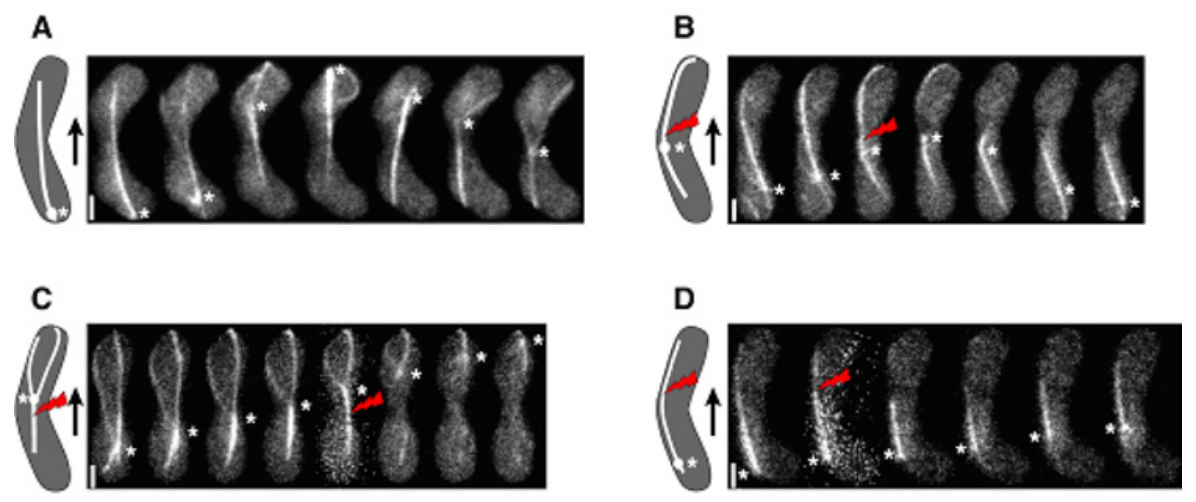

Fig. 5. Spindle pole body (SPB) movement is driven by pulling via the leading microtubules. (A-D) SPB oscillations in cells expressing Sid4-GFP (SPB marker, indicated by asterisks) and GFP-alpha2tubulin. Each panel shows selected images from a time-lapse sequence of a cell, together with a scheme of the SPB and microtubules at the time of laser ablation. The red bolt indicates the position of ablation; the black arrows indicate the direction of SPB movement. (SPB position as a function of time for Figs. 5(B)-5(D) are shown in Figs. 10(D)-10(F). (A) Control cell without laser ablation. (B) Laser ablation of the leading microtubules induced a change of direction of the SPB movement $(\mathrm{n}=19$ out of 21 cells; in the remaining 2 cells another microtubule pulled the SPB in the same direction). Laser ablation of the (C) trailing microtubules $(\mathrm{n}=19 / 19)$, or (D) the tip of the leading microtubules $(\mathrm{n}=$ 10/10) did not affect the SPB movement. Scale bars, $2 \mu \mathrm{m}$. Figure reproduced from Ref. [27].

We next asked whether the pulling force is generated at the interaction site between the leading microtubule tip and the cell end, or largely along the whole length of the leading microtubules [28]. To distinguish between these scenarios, we ablated the leading microtubules $\sim 4 \mu \mathrm{m}$ away from the microtubule tip. After the selective ablation of the microtubule tip region, the remaining $\sim 4 \mu \mathrm{m}$ long leading microtubules and the SPB continued to move forward (Fig. 5). This indicates that the force generated along the lateral microtubule-cortex interactions is large enough to generate the motion of the SPB observed during oscillation. Additional evidence for force generation along lateral microtubule-cortex interactions is provided by observations of SPB movement in the absence of interactions between the leading microtubules and the cell end in unperturbed cells [27].

\subsection{Dynein redistributes to the leading microtubules in response to the SPB velocity}

Evidence suggests that the minus-end directed motor cytoplasmic dynein [29] most likely generates the force for the SPB movement. Indeed, dynein heavy chain (Dhc1) deletion results in a lack of oscillations [17], even though meiotic divisions do still occur. Dynein, observed as a Dhc1-GFP, is localized at the SPB and microtubules [17].

In order to understand the interplay between dynein, microtubules, and the SPB movement, we quantified the dynamics of the spatial distribution of dynein during oscillations. We labelled Dhc1p with triple GFP and tubulin with mCherry [27]. We observed a strong dynein signal on the leading microtubules, and a weak signal on the trailing microtubules (Fig. 6). The signal on the leading microtubules was distributed in a spotted pattern, which was stationary with respect to the cortex (Fig. 6). The dynein dots disappeared after the SPB passed over their location. According to the measured force-velocity curve of dynein [30], a reverse motion, corresponding to the motion of the trailing microtubules, is associated with a high load on the motors. This suggests that the comparatively weak observed dynein signal on trailing microtubules is a consequence of load-dependent detachment of dynein.

We observed furthermore that after the dynein-mediated links between the microtubules and the cortex broke, dynein detached from the cortex and remained on the microtubules 

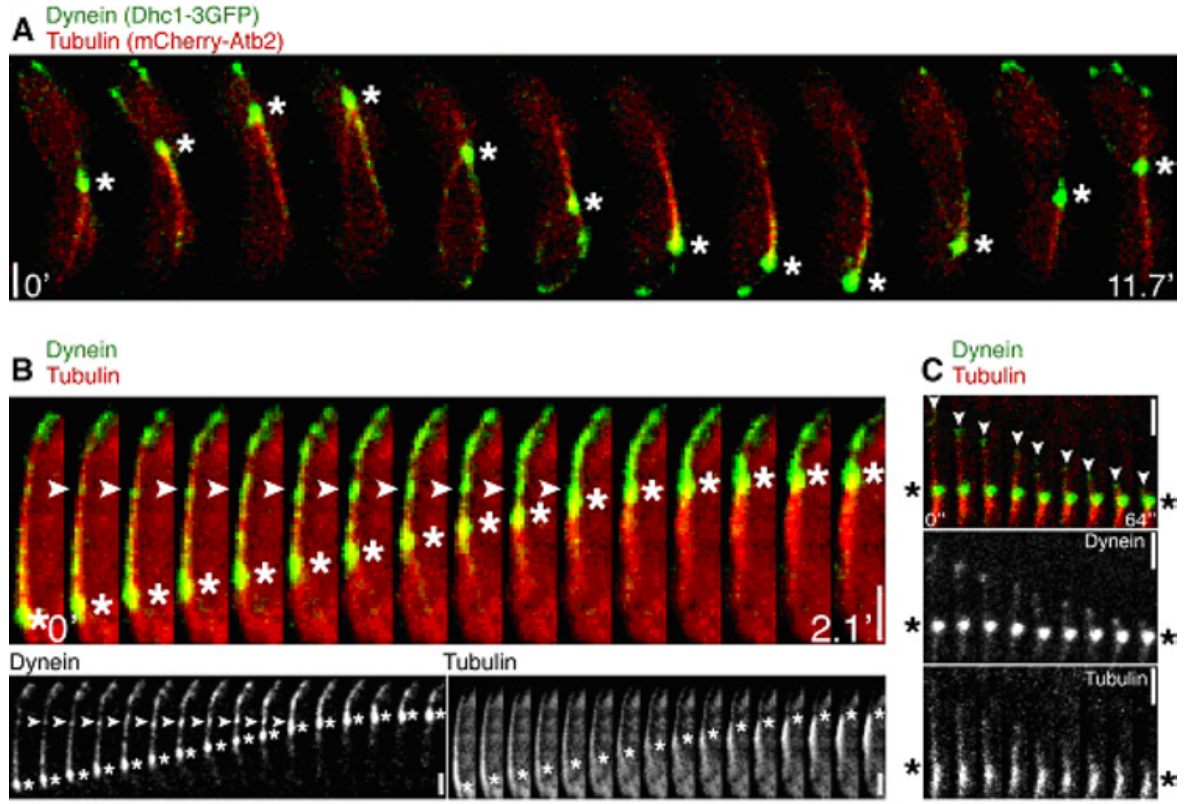

Fig. 6. The dynamics of dynein distributions. (A) A time-lapse sequence of a cell expressing Dhc1p3GFP (dynein; green) and mCherry-alpha2-tubulin (red) during one oscillation period. Dynein signal is stronger on the leading than on the trailing microtubules. (B) Time-lapse images of a region in the cell, showing that dynein is distributed in a spotted pattern along the leading microtubules. The dots are stationary with respect to the cell cortex (arrowheads). Dynein is removed from the cortex when the SPB passes over its location. Lower panels show GFP and mCherry channels separately. (C) Time-lapse images of a region in the cell, showing that when microtubules detach from the cortex, dynein also detaches from the cortex and remains on the plus-end of the depolymerizing microtubules (arrowheads). Lower panels show GFP and mCherry channels separately. Scale bars, $2 \mu \mathrm{m}$. Figure reproduced from Ref. [27].

(Figure 6). We thus conclude that dynein is more tightly bound to the microtubules than to the cortex. Current in vitro studies do not mimic this situation because such studies require that the motor tail domain is fixed to a bead or a surface $[16,30,31]$.

We next asked whether the observed spotted pattern of dynein (Figs. 6 and 7) represents dynein clusters along the lateral side or rather at the ends of individual microtubules. The tubulin signal along the leading microtubules was constant, suggesting that it resulted either from a single microtubule or from several microtubules of the same length (Fig. 7). To the contrary, the dynein signal showed several peaks along the same path (Fig. 7). We conclude that dynein is present along the microtubules and not only at their ends [27].

\subsection{The minimal model}

Our data suggest that dynein motors, anchored at the cell cortex along the leading microtubule, exert a pulling force on the microtubule, which pulls the SPB. But why does the SPB change direction and oscillate? There are three basic scenarios. First, there may be an underlying oscillation of cytoplasmic or cortical component(s), for example of the active cortical anchor proteins for dynein, which result in the observed oscillations of dynein, microtubules, and the SPB. Second, the SPB may provide the signal: when the SPB reaches one tip of the cell, it deactivates a cortical component required for force production (e.g. the cortical anchor proteins for dynein) in that region. The anchors are in the meantime re-activated at the other cell tip that is far away from the SPB, resulting in pulling towards that side [22]. Several lines of evidence 
A

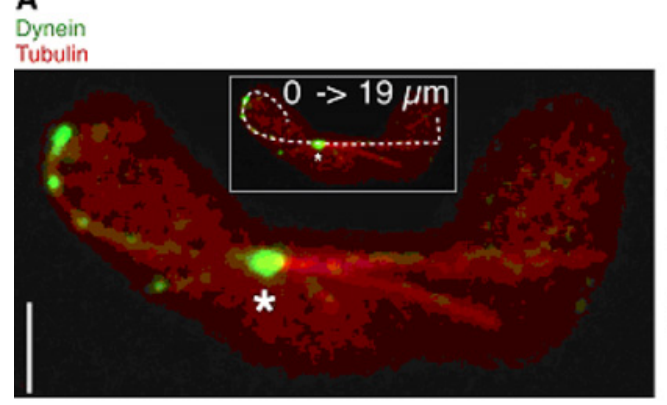

B

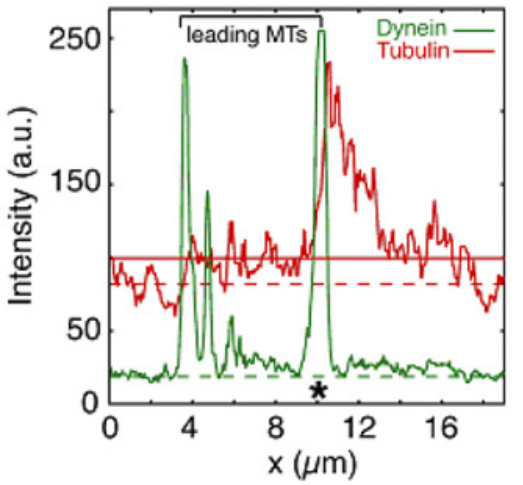

Fig. 7. Dynein is attached along the length of the microtubules. (A) Image and (B) the corresponding intensity profile of dynein (green) and tubulin (red) measured along the white dashed line demonstrate that dynein is present along the microtubules and not only at their ends. The estimated cytoplasmic signal intensity of dynein and tubulin is indicated by a dashed green and red line, respectively (B). The tubulin signal along the leading microtubules is relatively constant (solid red line), whereas the dynein signal (solid green line) shows several peaks along the same path (B). Asterisks indicate the position of the SPB. Scale bar, $2 \mu \mathrm{m}$. Figure reproduced from Ref. [27].

contradict both models. The leading MT can break at the end of the cell spontaneously due to extensive MT buckling, or as a result of laser ablation; in either case, breakage results in a short MT fragment which is free from the SPB. Since the SPB is near the same cell end as the fragment, the two proposed models predict that the force generators in this cell end are inactive and thus the microtubule fragments should not move. However, we have observed that the fragments slide along the cortex of the cell tip with a speed similar to the maximum speed of the SPB $(\sim 5 \mu \mathrm{m} / \mathrm{min})$. Furthermore, while the SPB is near the cell center, the leading microtubule occasionally detaches from the cell cortex, either spontaneously or induced by laser ablation. In this case, the models predict that in the subsequent $1 / 4$ of the period the SPB should remain stationary (in case of no further leading microtubule attachment to the cortex) or move forward (in case of leading microtubule re-attachment), because the force generators are active on the leading side. Yet, we observed quick changes of the SPB direction (within $<1 / 4$ of the period, Fig. 5).

In the third scenario, collective behavior of dynein motors acting on dynamic microtubules generates oscillations. The most striking observation in the Dhc-3GFP strain was that the dynein signal is strong on the leading microtubule and weak on the trailing microtubule, with an abrupt jump in intensity at the SPB. What makes the difference between the leading and the trailing microtubule? Let us consider three possibilities.

First, the age of the microtubule determines the amount of dynein attached to it. The assumption is that older microtubules accumulate more dynein. The leading microtubule is older that the trailing one, because the leading microtubule existed in the preceding end-toend movement as the trailing microtubule, whereas the current trailing microtubule is newly formed. A prediction that arises from this possibility is that dynein should accumulate on the trailing microtubule with time as the SPB moves forward. Our measurements showed a constant low Dhc-3GFP intensity along the trailing microtubule as the SPB moves, with an increase in intensity at the point when the SPB changes direction, thus, there is no time-dependent accumulation (Fig. 6). Moreover, observation of the plus-end tracking protein, Mal3 tagged with GFP, showed that individual new microtubules are constantly nucleated both forward and backward (with a bias in the backward direction during fast SPB movements), suggesting that the leading microtubule bundle is, on average, not older than the trailing one. Together, these data contradict the age-dependent model.

The second possibility is that the dynamic state of microtubules affects the attachment of dynein. Here, the assumption is that the shrinking microtubules have a stronger affinity for 
dynein compared to growing microtubules. The leading microtubule typically shrinks and may thus accumulate more dynein than the trailing one, which typically grows as the SPB moves forward. However, experiments in which microtubules were chemically depolymerized using MBC and then re-polymerized after MBC wash-out, as well as the strain with overexpressed Dhc1GFP, showed that growing microtubules can accumulate dynein. Furthermore, in a deletion strain without the cortical anchor proteins for dynein, we observed that the dynein signal on the microtubule decreases during microtubule shrinkage (data not shown). These observations contradict the dynamic state model.

The third possibility is: The different levels of dynein result from a difference in load experienced by the motors attached to the leading and the trailing microtubule. We developed a minimal one-dimensional description based on the experimental results described above and the known physical properties of motors, including their response to load [27]. We consider motors that attach to dynamic microtubules and link them to the cortex. The attachment rate depends on microtubule length and motor concentration; the detachment rate is load-dependent. The linked motors generate a force on the microtubules described by a force-velocity relationship.

This minimal model is represented in Fig. 8. Two microtubules extend from the SPB in opposite directions. Microtubules can be in one of two states: growing with velocity $v_{g}$ or shrinking with velocity $v_{s}$. The transition from growing to shrinking occurs when the plus end of a microtubule reaches the cell end. This assumption is based on experimental observations [22]. Subsequently, the microtubule shrinks until its length vanishes [22]; this is followed by nucleation of a growing microtubule. The viscous friction force and the forces $F_{l}$ and $F_{r}$ acting on the left and the right microtubule, are balanced,

$$
\xi \frac{\mathrm{d} x_{S P B}}{\mathrm{~d} t}=F_{l}+F_{r}
$$

where $x_{S P B}$ is the position of the SPB along the long axis of the cell, and $\xi$ is the friction coefficient of the system consisting of the nucleus, SPB, and microtubules [32]. The forces $F_{l}$ and $F_{r}$ are exerted by attached motors, $F_{l}=N_{l} f_{l}$ and $F_{r}=N_{r} f_{r}$. Here, $N_{l}$ and $N_{r}$ are the total number of motors attached to each microtubule and linked to the cortex, and $f_{l}$ and $f_{r}$ the forces generated by a single motor on the left and the right microtubule, respectively. The forces $f_{l}$ and $f_{r}$ are described by linear force-velocity relationships, $v=v_{0}\left(1+f_{l} / f_{0}\right)$ and $v=v_{0}\left(-1+f_{r} / f_{0}\right)$, respectively $[12,30,31,33]$. The velocity of the motor with respect to the microtubule is $v=-v_{S P B}=-\mathrm{d} x_{S P B} / \mathrm{d} t$, where $v_{S P B}$ is the SPB velocity. The velocity in the absence of force is denoted as $v_{0}$, and $f_{0}$ is the stall force of the motor. The length of the microtubule growing to the left and to the right is denoted $L_{l}$ and $L_{r}$, respectively. The linear densities, $n_{l}=N_{l} / L_{l}$ and $n_{r}=N_{r} / L_{r}$, of the motors attached to the left and the right microtubule, respectively, obey kinetic equations which describe the attachment and detachment of motors. For the right microtubule this equation reads:

$$
\frac{\mathrm{d} n_{r}}{\mathrm{~d} t}=k_{o n} c-k_{o f f}\left(f_{r}\right) n_{r}
$$

with $n_{r}=0$ at microtubule nucleation. Here, $c$ is the cytoplasmic concentration of motors, and $k_{\text {on }}$ characterizes the rate of attachment of motors to microtubules. As in Refs. $[12,15,34]$, the load-dependent motor detachment rate, $k_{o f f}$, is described by

$$
k_{o f f}\left(f_{r}\right)=k_{0} \exp \left(f_{r} / f_{c}\right) .
$$

Here, $f_{r}$ is the load force acting on individual motors, $k_{0}$ is the detachment rate in the absence of a load, and $f_{c}$ a characteristic force. The equations that describe the kinetics of motors on the left microtubule are obtained by substituting the subscript $r$ by $l$ in Eqs. (2) and (3), and changing the sign of the exponent in Eq. (3).

\subsection{Solutions of the minimal model}

Independent of the initial conditions, numerical solutions of the equations (1)-(3) attain limit cycles for typical parameter values (Fig. 8). The resulting oscillations of the SPB position have 


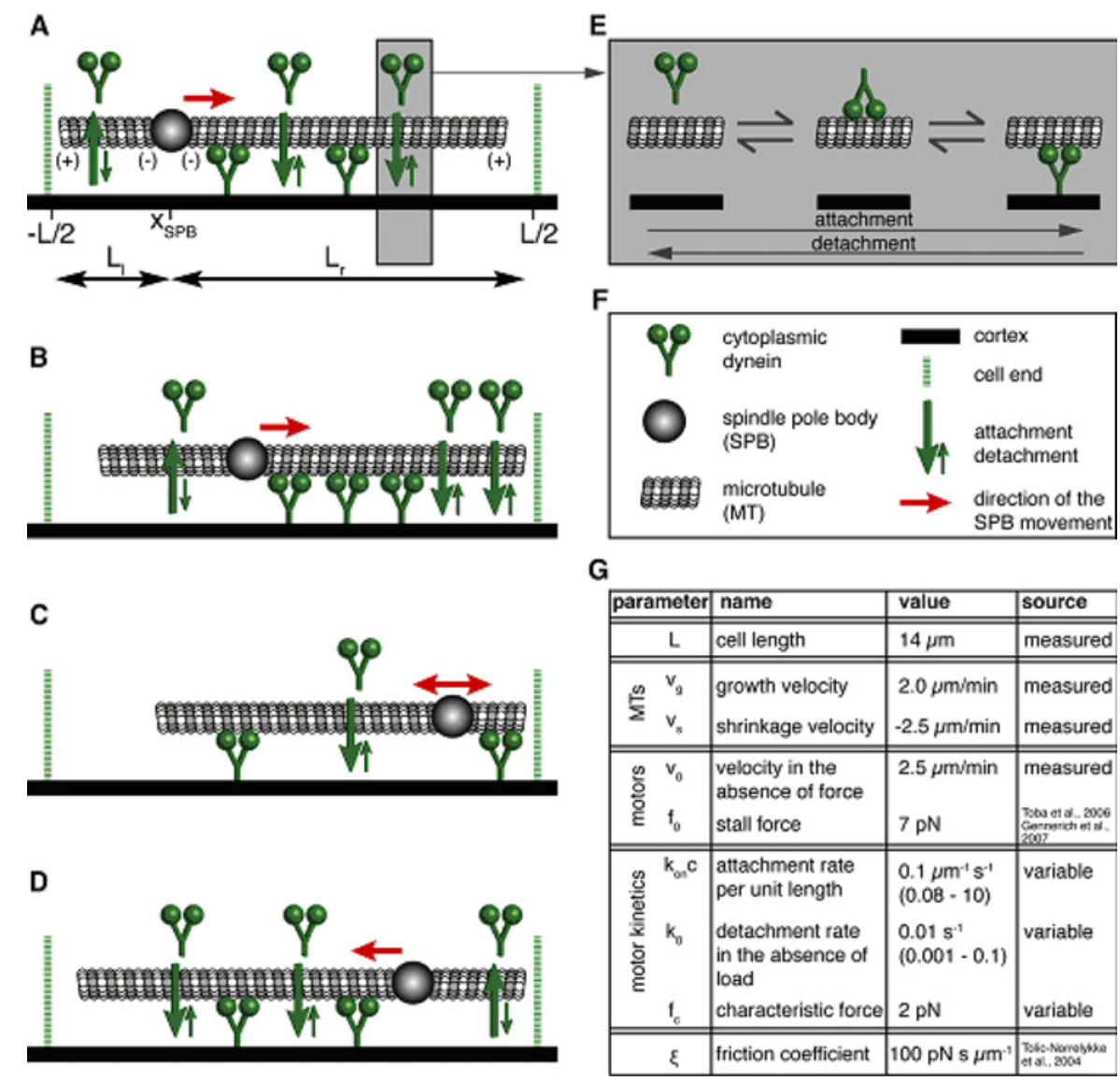

Fig. 8. The minimal model. (A-D) Schematic drawing of events during oscillations. (A) The position of the SPB along the longitudinal axis of the cell is denoted $x_{S P B}$. The two cell ends are located at $x=-L / 2$ and $x=L / 2$, respectively, where $L$ is the cell length. Two microtubules grow from the SPB. There are more motors attached to the microtubule on the right, thus the SPB moves to the right. As the SPB moves, the motors on the left microtubule are under high load, which stimulates their detachment. The load on the motors on the right microtubule is low. The asymmetry in the number of motors on the two microtubules grows, resulting in a faster SPB movement. (B) The faster movement further increases the asymmetry in the load on the motors, creating a positive feedback between the SPB movement and the number of motors. However, because of the finite size of the cell, the right microtubule shrinks and thus loses motors. (C) When the number of motors on the left and the right microtubule is equal, the SPB does not move. Since the left microtubule is longer than the right one, it accumulates more motors. (D) Thus, the SPB changes direction and the oscillation cycle continues. (E) Steps in the dynein attachment and detachment process; the intermediate step is not included in the model; (F) legend; $(\mathrm{G})$ parameters of the model; the values in brackets denote the intervals where the behavior of the model does not change. Figure reproduced from Ref. [27].

an almost triangular waveform, with an amplitude slightly smaller than $L / 2$ (Fig. $9(\mathrm{~A})$ ). This is reflected in the square waveform of the SPB velocity (Fig. 9(B)). The total number of dyneins attached to the microtubules depends on the SPB velocity: when the SPB starts to move, the number of dyneins attached to the leading microtubule increases (Fig. 9(C)). Simultaneously, the number of dyneins at the trailing microtubule decreases (Fig. 9(C)). The rapid detachment of dyneins from the trailing microtubule results from the high load force experienced by dyneins that oppose the SPB movement. The triangular waveform and the small number of dyneins on the trailing microtubule are observed in our minimal model in the regime of a strong dependence of the detachment rate on load $\left(f_{c}<\approx 4 \mathrm{pN}\right)$. In the regime of a weak load-dependence $\left(f_{c}>\approx\right.$ $10 \mathrm{pN})$, oscillations still occur but the waveform is close to sinusoidal and dynein accumulates on 


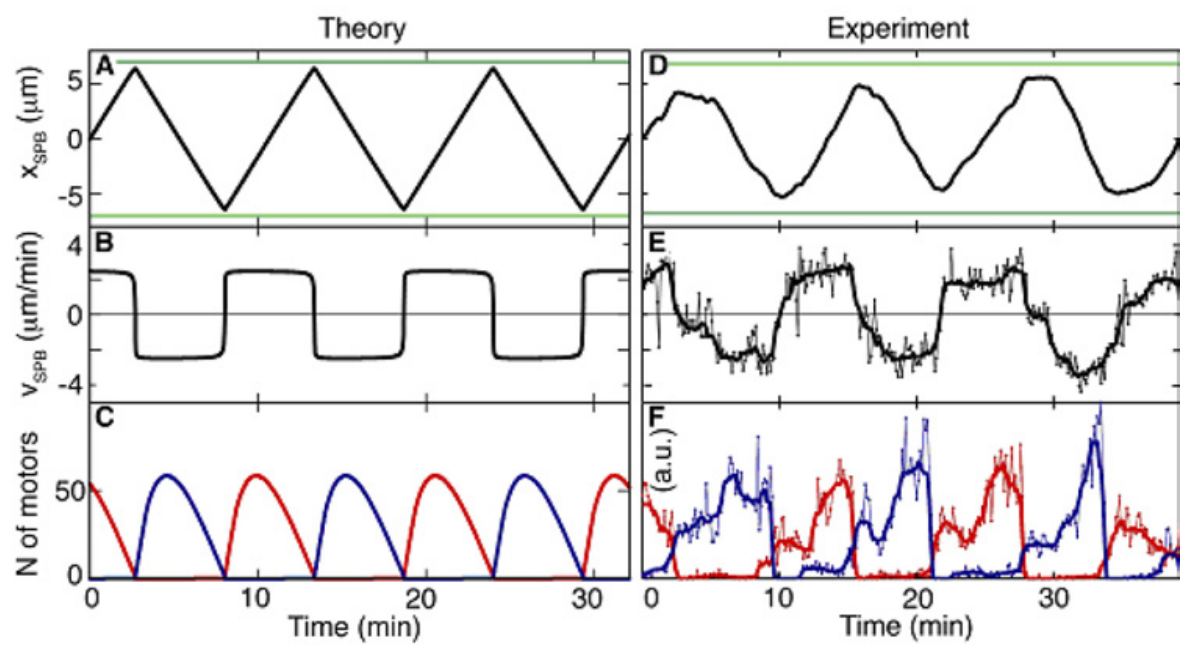

Fig. 9. Comparison of theory and experiments: oscillation pattern and the number of motors in unperturbed cells. (A-C) Numerical results and (D-F) experimental measurements of the time evolution of SPB position (A, D), SPB velocity (B, E), and the amount of dynein motors on the upper (red) and the lower (blue) microtubules $(\mathrm{C}, \mathrm{F})$. (E, F) Thin lines with markers show the original data, while the thick lines show the median in sliding windows of 10 data points. (C, F) When the SPB moves upwards $(v>0)$, the red curve shows the number of dyneins on the leading microtubule, while the blue curve the number on the trailing microtubule. When the SPB moves downwards $(v<0)$, the blue curve corresponds to the leading microtubule, and the red curve to the trailing microtubule. The numerical and the experimental results are plotted with the same scale (A and D, B and E), whereas the scaling factor between $\mathrm{C}$ and $\mathrm{F}$ is not known. The different shape of the curves in $\mathrm{C}$ and $\mathrm{F}$ is most likely due to the assumption of a uniform distribution of dynein along the microtubule in the model, whereas experiments showed a non-uniform distribution (Figs. 6 and 7). The numerical solutions (A-C) can be understood in a simple limit where the SPB moves with the maximal dynein velocity, $\mathrm{d} x_{S P B} / \mathrm{d} t= \pm v_{0}$. In this limit, oscillations have an exact triangular waveform. Furthermore, the dynein linear densities on the microtubules obey $\mathrm{d} n / \mathrm{d} t \approx k_{o n} c$ and $\mathrm{d} n / \mathrm{d} t \approx 0$, giving a parabolic dependence of the total number of dyneins at the leading microtubule with time, and small dynein numbers on the trailing microtubule, respectively (C). Figure reproduced from Ref. [27].

the trailing microtubule. In each of the two regimes, the waveform and the dynein distribution is robust for a large range of parameters of the minimal model.

If we generalize the minimal model to consider multiple microtubules at each side of the $\mathrm{SPB}$, as observed in experiments [22], the waveform and the dynein distribution do not change significantly in the strong load-dependence regime. By contrast, in the weak load-dependence regime, oscillations do not exist [27].

\subsection{Experimental tests of the model}

In order to determine the role of load-dependence in the observed oscillations, we compared the measured waveforms of the SPB position and velocity with those from theory, obtained in the regimes with strong and weak load-dependence. The measured waveforms of the SPB position and velocity are close to triangular and square waveforms, respectively (Figs. 9(D) and 9(E)). The experimental data match the theoretical prediction in the strong load-dependence regime (Figs. 9(A) and $9(\mathrm{~B})$ ). Consequently, in this regime the theory predicts a low number of motors on the trailing microtubule, due to a load-dependent detachment rate and redistribution of motors (Fig. 9(C)). Indeed, experiments independently showed the absence of dynein on the trailing microtubules (Fig. 9(F)). Moreover, the model accounts for the experimentally observed end-to-end SPB oscillations (Figs. $9(\mathrm{~A})$ and $9(\mathrm{D}))$ if the microtubule shrinkage velocity $\left(v_{s}\right)$ is comparable to the maximal motor velocity $\left(v_{0}\right)$. 

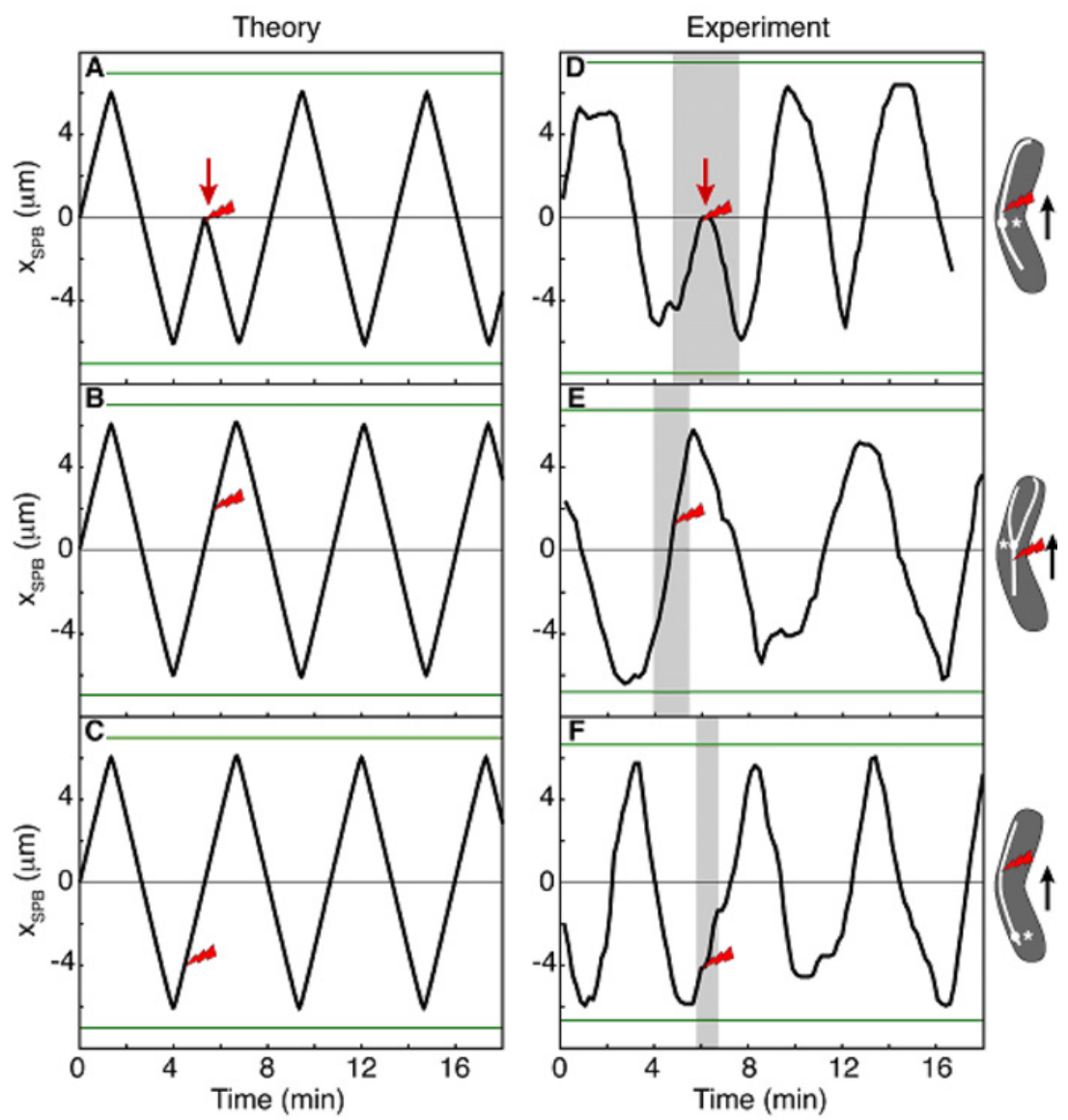

Fig. 10. Comparison of theory and experiments: oscillation pattern after microtubule cutting. (A-C) Numerical results and (D-F) experimental measurements of the SPB position as a function of time. The red bolt signs mark the time and the SPB position at the moment of microtubule cutting. Schemes show the SPB and the microtubules, as well as the site of laser ablation (bolt signs); the black arrows indicate the direction of SPB movement. Laser ablation of the leading microtubules induced a change of direction of the SPB movement (red arrows in A, D). In experiments, the change occurred within $25 \pm 13$ s after the ablation (mean \pm s.d., $n=12$ ). Laser ablation of the trailing microtubules $(\mathrm{B}, \mathrm{E})$ or the tip of the leading microtubules $(\mathrm{C}, \mathrm{F})$ did not perturb the SPB movement. The parameters in $(\mathrm{A}-\mathrm{C})$ are as in Fig. 8, except $v_{0}=5 \mu \mathrm{m} / \mathrm{min}, v_{s}=-5 \mu \mathrm{m} / \mathrm{min}$, and $v_{g}=3 \mu \mathrm{m} / \mathrm{min}$. The gray rectangles in (D-F) mark the time interval of the images shown in Figs. 5(B)-5(D); the green lines mark the cell ends. Figure reproduced from Ref. [27].

The SPB movement can be perturbed by laser-cutting of microtubules (Fig. 5). Laser-cutting of the leading microtubule close to the SPB resulted in a rapid reversal of the SPB movement (Figs. 5(B) and 10(D)). To build the model, we used the conclusion that SPB movement is driven by microtubule pulling. The same experiment, however, provides additional information such as the rapid reversal of the SPB movement, which allows for testing of the model. To test the predictive power of our model we simulated the cutting experiment in the model by setting the length of the leading microtubule to zero instantaneously when the SPB was close to the center. This mimicked the experimental situation after laser cutting, where the length of the leading microtubule was below the resolution of the microscope. After the disappearance of the leading microtubule in the simulations, the SPB quickly changed the direction of motion (Fig. 10(A)), in agreement with the experiments (Fig. 10(D)).

For completeness, we tested whether the model is able to reproduce the remaining two cutting experiments (Figs. 5(C) and 5(D)). In our experiment, laser-cutting of the trailing 
microtubules close to the SPB, or cutting of the leading microtubules $\sim 4 \mu \mathrm{m}$ away from the SPB, did not perturb the SPB movement (Figs. 5(C), 5(D), 10(E), 10(F)). Consistent with our observation, mimicking the laser-cutting of the trailing microtubules in the model by instantaneously setting the length of the trailing microtubule to zero did not affect the SPB movement (Fig. 10(B)). Similarly, the SPB movement remained unchanged when the length of the leading microtubule was instantaneously decreased to $4 \mu \mathrm{m}$, after which the microtubule resumed growth (Fig. 10(C)). Thus, the behavior of the model is consistent with microtubule cutting experiments.

While our model captures nearly all aspects of the observed behavior of the nuclear oscillations, it does not account for details such as the frequent SPB pausing at the cell end (denoted Phase II in Ref. [22]; see also Fig. 9(D)), and dynein accumulation in dots and at the SPB [22] (Figs. 6 and 7). Future work will explore these observations.

\section{Discussion}

The mechanism we propose for the meiotic nuclear oscillations in $S$. pombe is based on selforganization of dynein motors, without a requirement for additional spatio-temporal regulation. The self-organization of motors in this system occurs through load-dependent detachment of dynein motors and their redistribution via the cytoplasm. From the cytoplasm, motors attach to microtubules in a microtubule length-dependent manner; this asymmetry causes the switch in the direction of motion necessary for the oscillatory behavior.

\subsection{Load-dependent detachment of dynein motors}

The probability of breaking a bond between two objects, such as between a motor protein and a microtubule, is increased by an external pulling force [34,35]. This process is known as load-dependent detachment. Detachment of motors from microtubules in response to load forces has been studied in vitro [16] and a theoretical description has been developed [36]. Load-dependent detachment of motor proteins has been suggested to play a crucial role in bidirectional transport processes as well as spindle and chromosome oscillations $[10,12,14,15]$. However, how motor proteins respond to load forces in vivo is not understood.

In general, a microtubule motor protein either walks to the plus end or to the minus end of a microtubule (depending on the motor type), and the walking velocity depends on the load force exerted on the motor. As the resisting load force increases, the motor velocity decreases; when the load exceeds a certain value the motor will move in the opposite direction. If the tail of the motor is fixed to a surface and its head attached to a microtubule, the direction and the velocity of the motor can be determined from the movement of the microtubule. Hence, the load force on the motor depends on the movement of the microtubule.

During the meiotic nuclear oscillations dynein motors link microtubules to the cell cortex. The microtubules extend from the SPB in one or a few bundles pointing with their plus ends towards each cell end. For simplicity, we consider only one microtubule extending in each direction. Based on this geometry, we define two populations of dynein motors that are bound to oppositely oriented microtubules: one population walks toward the minus end of the microtubules while the other is forced, by the microtubule movement, to walk towards the plus end of the microtubules. Therefore, during the SPB movement, the dynein population forced to walk towards the plus end of the microtubules is under higher load force and has a higher probability to detach from the microtubules.

In our study, we imaged dynein motors during the oscillations and observed an asymmetry in the motor distribution. Dynein motors, which would oppose the SPB movement, are visible neither at the cortex nor at the microtubules. We conclude that dynein detaches from the microtubule and the cortex in response to load forces. To our knowledge, this is the first in vivo visualization of load-dependent dynein detachment. We propose that dynein does not detach simultaneously from the cortex and from the microtubule. Based on the observation that dynein remains on the plus end of the microtubule after the release of the plus end from the cortex, we 
conclude that dynein first detaches from the cortex and then from the microtubule. This differs from scenarios where force generators detach from microtubule ends but remain localized on the cell cortex $[12,15]$ or chromosomes [10].

\subsection{Redistribution of dynein motors via the cytoplasm}

Redistribution of proteins is crucial for a variety of physiological processes. During nuclear oscillations, dyneins detach from the cortex in response to higher load forces and accumulate to sites where they experience less load forces, which is the side with more dyneins. This redistribution is largely independent of microtubule length, implying that shorter microtubules can lead the movement by accumulating more motors than longer microtubules. Pulling by shorter microtubules is observed experimentally and is a prerequisite for the oscillations. Dynein redistribution is possible because dyneins detach both from the microtubules and from the cell cortex.

\subsection{Microtubule length-dependent motor attachment}

Dynein motors from the cytoplasm can attach along the length of microtubules and the number of attachment sites depends on the length of the microtubules. Attachment of dynein along the length of the microtubules is confirmed directly by imaging dynein motors and indirectly by laser ablation.

During the meiotic oscillations, length-dependent attachment of dynein is necessary for the change of direction of the SPB movement. When the movement of the SPB stops near a cell end, the microtubules extending towards the opposite cell end are longer than those pointing to the proximal cell end. Due to the length-dependent attachment of dynein, more motors will accumulate on the longer microtubules. Thus, a movement starts in the direction of the longer microtubules. Once the movement has started, the motors on the longer microtubules experience lower load forces, the motor detachment rate thus decreases and the motors accumulate on the longer microtubules, thereby the change of direction is completed. After the change of direction is completed, the microtubule length does not play a key role in dynein accumulation and the resulting force. This mechanism differs from those where the pulling force depends on the length of the microtubule $[28,37]$.

\section{Conclusion}

Our work emphasizes that self-organized collective behavior of motors acting on dynamic microtubules can account for large-scale movements of the nucleus during meiosis. We demonstrate that load-dependent detachment of motors occurs in vivo, and that it introduces the dynamic instability necessary for the oscillations. Furthermore, we show that dynein motors are more strongly attached to microtubules than to the cell cortex, which differs from scenarios where motors are assumed to be fixed at the cell cortex and detach from microtubules $[10,12,15]$. In response to load forces, dynein motors dynamically redistribute in the cell and attach to the microtubules extending in front of the moving SPB, thereby generating oscillations. Dynamic protein distributions in cells are traditionally interpreted in terms of reaction-diffusion mechanisms [38]. Here, we have shown that load-dependent collective action of motor proteins can generate spatio-temporal pattern by a very different mechanism.

We thank Y. Hiraoka, G. Steinberg, T. Toda, M. Yamamoto, J. Bähler, K. Tanaka, K. Gould, T.D. Pollard, and R. Tsien for strains and plasmids; D. Accardi for taking the images of mating cells; J. Peychl, B. Schroth-Diez, I. Raabe, A. Oates, M. Boes, I. Šarić, H. Kahl, and R. Hobson for technical support, and J. Howard, C. Leduc, S.W. Grill, S. Diez, E. Paluch, A.A. Hyman, T. Gross, K.E. Sawin, V. Krstić, and the members of the Tolić-Nørrelykke group for discussions. 


\section{References}

1. S. Busson, D. Dujardin, A. Moreau, J. Dompierre, J.R. De Mey, Curr. Biol. 8, 541 (1998)

2. D. Eshel, L.A. Urrestarazu, S. Vissers, J.C. Jauniaux, J. C. van Vliet-Reedijk, R.J. Planta, I.R. Gibbons, Proc. Natl. Acad. Sci. USA 90, 11172 (1993)

3. Y.Y. Li, E. Yeh, T. Hays, K. Bloom, Proc. Natl. Acad. Sci. USA 90, 10096 (1993)

4. A.R. Skop, J.G. White, Curr. Biol. 8, 1110 (1998)

5. J. Howard, Mechanics of Motor Proteins and the Cytoskeleton Sunderland MA Sinauer Associates (2001)

6. A.S. Bajer, J. Cell Biol. 93, 33 (1982)

7. Y. Chikashige, D.Q. Ding, H. Funabiki, T. Haraguchi, S. Mashiko, M. Yanagida, Y. Hiraoka, Science 264, 270 (1994)

8. G. Fink, I. Schuchardt, J. Colombelli, E. Stelzer, G. Steinberg, Embo. J. 25, 4897 (2006)

9. C.L. Rieder, E.A. Davison, L.C. Jensen, L. Cassimeris, E.D. Salmon, J. Cell Biol. 103, 581 (1986)

10. O. Campas, P. Sens, Phys. Rev. Lett. 97, 128102 (2006)

11. G. Civelekoglu-Scholey, D.J. Sharp, A. Mogilner, J.M. Scholey, Biophys. J. 90, 3966 (2006)

12. S.W. Grill, K. Kruse, F. Julicher, Phys. Rev. Lett. 94, 108104 (2005)

13. A.P. Joglekar, A.J. Hunt, Biophys. J. 83, 42 (2002)

14. C. Kozlowski, M. Srayko, F. Nedelec, Cell 129, 499 (2007)

15. J. Pecreaux, J.C. Roper, K. Kruse, F. Julicher, A.A. Hyman, S.W. Grill, J. Howard, Curr. Biol. 16, $2111(2006)$

16. C.M. Coppin, D.W. Pierce, L. Hsu, R.D. Vale, Proc. Natl. Acad. Sci. USA 94, 8539 (1997)

17. A. Yamamoto, R.R. West, J.R. McIntosh, Y. Hiraoka, J. Cell Biol. 145, 1233 (1999)

18. D.Q. Ding, A. Yamamoto, T. Haraguchi, Y. Hiraoka, Dev. Cell, 6, 329 (2004)

19. M. Parvinen, K.O. Soderstrom, Nature 260, 534 (1976)

20. E. Trelles-Sticken, C. Adelfalk, J. Loidl, H. Scherthan, J. Cell Biol. 170, 213 (2005)

21. D.Q. Ding, Y. Chikashige, T. Haraguchi, Y. Hiraoka, J. Cell. Sci. 111, 701 (1998)

22. A. Yamamoto, C. Tsutsumi, H. Kojima, K. Oiwa, Y. Hiraoka, Mol. Biol. Cell. 12, 3933 (2001)

23. N. Maghelli, I.M. Tolic-Norrelykke, J. Biophotonics 1, 299 (2008)

24. H. Scherthan, H. Wang, C. Adelfalk, E.J. White, C. Cowan, W.Z. Cande, D.B. Kaback, Proc. Natl. Acad. Sci. USA 104, 16934 (2007)

25. L. Sacconi, I.M. Tolic-Norrelykke, R. Antolini, F.S. Pavone, J. Biomed. Opt. 10, 14002 (2005)

26. I.M. Tolic-Norrelykke, L. Sacconi, G. Thon, F.S. Pavone, Curr. Biol. 14, 1181 (2004)

27. S.K. Vogel, N. Pavin, N. Maghelli, F. Julicher, I.M. Tolic-Norrelykke, PLoS Biol. 7, e1000087 (2009)

28. M.S. Hamaguchi, Y. Hiramoto, Dev. Growth Differ. 28, 143 (1986)

29. B.M. Paschal, H.S. Shpetner, R.B. Vallee, J. Cell Biol. 105, 1273 (1987)

30. A. Gennerich, A.P. Carter, S.L. Reck-Peterson, R.D. Vale, Cell 131, 952 (2007)

31. S. Toba, T.M. Watanabe, L. Yamaguchi-Okimoto, Y.Y. Toyoshima, H. Higuchi, H., Proc. Natl. Acad. Sci. USA 103, 5741 (2006)

32. I.M. Tolic-Norrelykke, E.L. Munteanu, G. Thon, L. Oddershede, K. Berg-Sorensen, Phys. Rev. Lett. 93, 078102 (2004)

33. F. Julicher, A. Ajdari, J. Prost, Rev. Mod. Phys. 69, 1269 (1997)

34. G.I. Bell, Science 200, 618 (1978)

35. E. Evans, Ann. Rev. Biophys. Biomol. Struct. 30, 105 (2001)

36. A. Parmeggiani, F. Juelicher, L. Peliti, J. Prost, Europhys. Lett. 56, 603 (2001)

37. A. Kimura, S. Onami, Dev. Cell 8, 765 (2005)

38. A.M. Turing, Bull. Math. Biol. 52, 153 (1990); discussion 119 